\title{
On the choice of regularization parameters in specification testing: a critical discussion
}

\author{
Stefan Sperlich
}

Received: 28 March 2011 / Accepted: 23 March 2013 / Published online: 20 November 2013

(C) Springer-Verlag Berlin Heidelberg 2013

\begin{abstract}
This article reviews and discusses the problem of choosing smoothing parameters and resampling schemes for specification tests in econometrics. While smoothing is used for the regularization of the non-specified parts of the null hypothesis and omnibus alternatives, the resampling serves for determining the critical value. Several of the existing selection methods are discussed, implemented, and compared. This has been done for cross-sectional data along the example of additivity testing. Doubtless, all problems considered here carry over to specification testing with dependent data. Intensive simulations illustrate that this is still an open problem that easily corrupts these tests in practice. Possible ways out of the dilemma are proposed.
\end{abstract}

Keywords Nonparametric specification tests - Adaptive testing - Bandwidth choice · Bootstrap · Subsampling

JEL Classification $\mathrm{C} 12 \cdot \mathrm{C} 14 \cdot \mathrm{C} 52$

\section{Introduction}

A decade after the review book of Hart (1997), non- and semiparametric specification testing is still quite a popular research field, especially in econometric theory. Any Internet search engine finds several hundred papers dealing with this topic even when limiting the search to the last 5 years. While the choice of appropriate smoothing parameters is fundamental for these tests, only a few articles address this issue. The Journal of Econometrics published in 2008 is a special issue devoted exclusively to

S. Sperlich $(\varangle)$

Département des sciences économiques and Research Center for Statistics,

Université de Genève, Bd du Pont d'Arve 40, 1211 Geneva, Switzerland

e-mail: stefan.sperlich@unige.ch 
semi- and nonparametric testing. Only in Gao et al. (2008) was the smoothing parameter choice explicitly considered - unavoidably, because they considered structural breaks. Moreover, for testing, these choice problems are not equivalent to the ones in regression. For example, it is well known that the optimal smoothing parameters for testing have different rates from those which are optimal for estimation; Ibragimov and Khasminski (1981) and Ingster (1982, 1993) are the classic references.

In the last couple of years, there has been a growing amount of literature on adaptive testing. This "adaptiveness" refers to the unknown smoothness of the alternative and therefore deals with the choice of smoothing parameters for the test statistic; see, e.g., Ledwina (1994), Kulasekera and Wang (1997), Spokoiny (1998), Kallenberg and Ledwina (1995), Horowitz and Spokoiny (2001), and Guerre and Lavergne (2005). Even though these methods have had little impact in the sense that we could not find published papers using them, they have been useful for a better understanding of the problem. Most of these papers concentrate on testing problems where the null hypothesis is fully parametric. Already Hart (1997) and later Eubank et al. (2005) discussed for those cases the problem of bandwidth choice in nonparametric specification testing; see also Zhang $(2003,2004)$ for a related discussion. Among others, Gao and Gijbels (2008) extensively discussed the role of bandwidth selection in kernel testing when the null is parametric. In that case, it is clear that the testing bandwidth is just a nuisance parameter which may not even be identified when size and power of the test are the main focus. It is not clear to what extend the proposed methods will help if the null hypothesis is semi- or nonparametric. However, this is not that rare; even additivity (or more general "separability") tests belong to this family. Other popular examples are tests for particular covariance structures including independence, tests for symmetry of distributions or substitution matrices, tests for profit maximization, etc.

Where a semi- or nonparametric null hypothesis has to be estimated explicitly, an additional smoothing parameter is needed. In many cases, this is chosen by crossvalidation or simply ad hoc without further justification. When bootstrap is used to determine the critical value, these tests entail another parameter choice problem: for pre-estimating the model under the null hypothesis to later generate the bootstrap samples. In most cases, the bandwidths for estimation and the bootstrap must have different rates; see Härdle and Marron (1991), González-Manteiga et al. (2004), or Cao-Abad and González-Manteiga (1993). Although these authors have already mentioned the problem of choosing an appropriate bandwidth, in practical applications, this problem has hardly been addressed. As a consequence, in most published procedures for testing or constructing confidence bands with a semi- or nonparametric null hypothesis, there is no guarantee that the test would hold the level, or the bands the nominal coverage probability; see for example Dette et al. (2005) or Rodriguez-Póo et al. (2012). However, in the former, it is not referred to as a bandwidth problem, but rather as a problem of design and dimensionality because the size distortion is much smaller for covariates being independent among each other. In the latter paper, the problem is avoided by using subsampling instead of bootstrap. It should be mentioned that there, the authors can even use a semiparametric bootstrap drawing the bootstrap errors from a distribution known up to one parameter. Although that parameter depends on further nonparametric nuisance parameters, the knowledge of distribution greatly mitigates the impact of bandwidths on the estimates of critical values. 
Su and White (2010) test for structural changes in partially linear models for time series. They need an "undersmoothing" bandwidth for the original test; the bandwidth choice for the bootstrap is not discussed in detail. In Su and Ullah (2013), a Zheng (1996)-type statistic is used to test for conditional heteroscedasticity. For the testing bandwidth, they refer to Horowitz and Spokoiny (2001) and Chen and Gao (2007), but thanks to a parametric mean function, they do not need another one for generating bootstrap samples. Su et al. (2013) test for additive separability of the error term in nonparametric structural models. They succeed in constructing a test statistic that compares a constant (for the null hypothesis) versus nonparametric densities (for the alternatives). As a bandwidth selector, they propose then Silverman's rule of thumb. The bandwidth problem for the there-applied smoothed bootstrap (cf. Cao-Abad and González-Manteiga 1993 or Cao-Abad 1993) has not been further studied. In Su et al. (2013), functional coefficient IV models are tested for constant coefficients. While a cross-validation bandwidth is proposed for the estimation of the alternative (which asymptotically is suboptimal for testing, see discussion above), they recommend the usage of a special fixed design bootstrap based on a purely parametric regression model and therefore different from the classic wild bootstrap. This is done as the authors were aware of the problem of a necessary oversmoothing for the latter. For more discussion and examples of nonparametric specification tests, see the comprehensive review of González-Manteiga and Crujeiras (2013). However, they only briefly comment on the problem of bandwidth choice in the context of calibration and optimal convergence rates.

To illustrate the outlined problem, we concentrate on the testing for additivity in cross-sectional data. We limit the presentation to statistics considered in Dette et al. (2005); Rodriguez-Póo et al. (2012), and the methods of Horowitz and Spokoiny (2001), Guerre and Lavergne (2005), and Gao and Gijbels (2008). The aim is not to find the most efficient additivity test or to propose new ones. A discussion of what are reasonable test statistics can be found in Roca-Pardiñas and Sperlich (2007). Here, our focus is only directed at a reasonable choice of regularization parameters that hold the level and guarantee non-trivial power.

In the next section, we review the estimation and testing procedures used for illustration. In Sect. 3, we discuss the different scenarios from which the practitioner has to make his choice, including modifications of test statistics, and resampling method. Section 4 summarizes the main findings from our simulations, and Sect. 5 concludes the paper. GAUSS codes, used for the simulations, are available from the author upon request.

\section{Model, estimators, and test statistics}

Before we introduce estimators and test statistics for a $d$-dimensional regression problem, let us briefly discuss the consequences of implicit conventions or explicit conditions you find in almost all related articles. Often the tests are analyzed in detail only for the univariate case, indicating that the extension to the multivariate one is straight. There, optimal smoothing requires the use of a bandwidth matrix, say $\mathbf{H} \in \mathbb{R}^{d \times d}$, which transforms the whole data matrix unless it is restricted to being diagonal. Even 
when abstracting from practical problems of determining a complete $\mathbf{H}$, such crosscovariate transformations are often undesirable for reasons of interpretability, especially for separable models. Presumably for that reason, it is mainly just in the context of density estimation where suggestions for the choice of $\mathbf{H}$ can be found. A most natural transformation is to turn all variables toward orthogonality and to normalize them afterward; see Duong and Hazelton (2003) for details. Intuitively, one might conclude that this is also recommendable for multivariate regression, but this can hardly be seen from the asymptotics. Actually, we are not aware of a study that explicitly considers a practical selector for $\mathbf{H}$ in multivariate regression containing off-diagonals. Furthermore, for convenience, it is typically assumed that the density of covariates (and the regression function) has the same smoothness in all directions, and that all covariates have either the same variance or the same support. Having done so, $\mathbf{H}$ gets reduced to $h \cdot \mathbf{I}_{d}$. In practice, one therefore divides each covariate by its standard deviation and then applies the same bandwidth $h$ to all. In order to concentrate on the various selection problems emerging in the testing context, we follow this custom, being aware that this is just a makeshift. For certain additive model estimators, some authors proposed methods which allow for different diagonal elements of $\mathbf{H}$.

\subsection{Model and estimation}

Consider the following general regression model:

$$
Y_{i}=m\left(X_{i}\right)+u_{i} \quad i=1,2, \ldots, n,
$$

with $\left\{\left(X_{i}, Y_{i}\right)\right\}_{i=1}^{n} \in \mathbb{R}^{d} \times \mathbb{R}$ i.i.d. (for simplicity), $m: \mathbb{R}^{d} \rightarrow \mathbb{R}$ the unknown function of interest, $m(x)=E(Y \mid X=x)$, and $u_{i}$ random errors with $E\left[u_{i}\right]=E\left[u_{i} \mid x_{i}\right]=0$ and finite variance $\sigma^{2}\left(x_{i}\right)$. The "internalized" (cf. Jones et al. (1994)) version of the Nadaraya-Watson estimator is defined as

$$
\hat{m}(x)=\frac{1}{n} \sum_{i=1}^{n} Y_{i}\left(\hat{f}_{k}\left(X_{i}\right)\right)^{-1} \mathbf{K}_{k}\left(x-X_{i}\right),
$$

where $\hat{f}_{k}\left(X_{i}\right)=\frac{1}{n} \sum_{j=1}^{n} \mathbf{K}_{k}\left(X_{i}-X_{j}\right)$ is a kernel density estimator, $\mathbf{K}_{k}\left(v_{1}, \ldots, v_{d}\right)=$ $\prod_{\delta=1}^{d} K_{k}\left(v_{\delta}\right)$ a product kernel with $K_{k}\left(v_{\delta}\right)=k^{-1} K\left(v_{\delta} k^{-1}\right)$ for $\delta=1, \ldots, d$. For the ease of presentation, we follow the common line of applying the same bandwidth $k$ to all covariates neglecting the possibility of using bandwidth matrices. As said, all covariates should then be brought to the same scale or range. Commonly, the kernel is assumed to be Lipschitz continuous with compact support and $\int|K(v)| d v<$ $\infty, \int K(v) d v=1$. Furthermore, $k$ is a bandwidth assumed to tend to zero for sample size $n$ going to infinity, but $n k^{d}$ going to infinity. Let us call $\hat{m}$ the estimator for the alternative; $k$ can then be considered as the "testing bandwidth."

Assume we are interested in additive modeling. We write

$$
E(Y \mid X=x)=m_{S}(x)=\psi+\sum_{\delta=1}^{d} m_{\delta}\left(x_{\delta}\right),
$$


setting $E_{X_{\delta}}\left\{m_{\delta}\left(X_{\delta}\right)\right\}=\int m_{\delta}(x) f_{\delta}(x) d x=0$ for all covariates $X_{\delta}$ for identification. Here, $m_{\delta}, \delta=1, \ldots, d$ are the marginal impact functions of each regressor. Therefore, $\psi$ is a constant equal to the unconditional expectation of $Y$. Writing $m(X)=$ $\psi+m_{\delta}\left(X_{\delta}\right)+m_{-\delta}\left(X_{-\delta}\right)$ where $X_{-\delta}$ is the vector $X$ of all explanatory variables without $X_{\delta}$, i.e., $X_{-\delta}=\left(X_{i 1}, \ldots, X_{i(\delta-1)}, X_{i(\delta+1)}, \ldots, X_{i d}\right)$ the identification condition directly induces an estimator for $m_{\delta}$. There exist different motivations to obtain

$$
\hat{m}_{\delta}\left(x_{\delta}\right)=\frac{1}{n} \sum_{i=1}^{n} Y_{i} K_{h}\left(x_{\delta}-X_{i \delta}\right) \frac{\hat{f}_{-\delta}\left(X_{i,-\delta}\right)}{\hat{f}\left(X_{i \delta}, X_{i,-\delta}\right)}
$$

like the argument of Kim et al. (1999), saying that there exist kernel weights $\omega\left(X_{\delta}, X_{-\delta}\right)$ so that $E\left[\omega\left(X_{\delta}, X_{-\delta}\right) Y \mid X_{\delta}=x_{\delta}\right]=\psi+m_{\delta}\left(x_{\delta}\right)$. Finally, with $\hat{\psi}=\frac{1}{n} \sum_{i=1}^{n} Y_{i}$ one sets $\hat{m}_{S}\left(X_{j}\right)=\hat{\psi}+\sum_{\delta=1}^{d} \hat{m}_{\delta}\left(X_{j \delta}\right)$ for each $j=1,2, \ldots, n$. The densities in (10) are kernel densities with bandwidths $h$ for variable $X_{\delta}$ and $h_{-\delta}$ else; see Dette et al. (2005) or Hengartner and Sperlich (2005) for details. In the following, (10) will be the estimator for the null hypothesis with bandwidth $h$ (and $h_{-\delta}$ ). As for $k$, we neglect bandwidth matrices and use the same bandwidth for all covariates supposing they have comparable distributions; recall our discussion from above.

For completeness, we conclude this subsection with a brief, non-complete review of available alternative estimators for nonparametric additive models. The probably first key reference is the classical backfitting estimator of Hastie and Tibshirani (1990). The lack of asymptotic theory for this estimator led to alternative proposals like the marginal integration estimator of Linton and Nielsen (1995), and its improved version of Kim et al. (1999) which we are using here. Comparison studies were provided by Sperlich et al. (1999) and Dette et al. (2005). About the same time appeared the smooth backfitting estimator of Mammen et al. (1999). Today, penalized spline versions of the classical backfitting are very popular in practice due to its easy implementation; see Fahrmeir et al. (2004) and Wood (2008).

\subsection{Test statistics}

The null hypothesis of interest is $H_{0}: m(\cdot)=m_{S}(\cdot)$ versus $H_{1}: m(\cdot) \neq m_{S}(\cdot)$. We do not aim to introduce new testing procedures, but rather take statistics which have already been studied in Dette et al. (2005) together with other additivity tests, motivated by Rodriguez-Póo et al. (2012), and which performed excellently in the study of Roca-Pardiñas and Sperlich (2007). Finally, we add a new test for additivity which is based on the statistic introduced in Guerre and Lavergne (2005). For more details about the tests, the reader is referred to the original papers. Consider the test statistics

$$
\begin{aligned}
\tau_{1} & =\frac{1}{n} \sum_{i=1}^{n}\left(\hat{m}\left(X_{i}\right)-\hat{m}_{S}\left(X_{i}\right)\right)^{2} w\left(X_{i}\right), \\
\tau_{2} & =\frac{1}{n} \sum_{i=1}^{n} \hat{e}_{i}\left(\hat{m}\left(X_{i}\right)-\hat{m}_{S}\left(X_{i}\right)\right) w\left(X_{i}\right),
\end{aligned}
$$


where $\hat{e}_{i}=Y_{i}-\hat{m}_{S}\left(X_{i}\right)$, i.e., the residuals under the null hypothesis, and $w(\cdot)$ an optional weight function that might be used for trimming. While $\tau_{1}$ calculates directly the integrated squared difference between the null and alternative models, $\tau_{2}$ seeks to mitigate the bias problem inherited from the estimate $\hat{m}$ which suffers from the curse of dimensionality. While in $\tau_{1}$ this large bias enters squared, in $\tau_{2}$ it only appears in simple terms multiplied by residuals which under $H_{0}$ should even be independent (almost, they are related to the smoothing bias) of the bias. Therefore, using leave- $\left(Y_{i}, X_{i}\right)$-out estimators for $\hat{m}_{S}\left(X_{i}\right)$ and $\hat{m}\left(X_{i}\right)$ in $\tau_{2}$ would give $E_{H_{0}}\left\{\tau_{2}\right\}=0$ asymptotically, see Gozalo and Linton (2001). Else, in Dette et al. (2005), it has been proved that each $n k^{\frac{d}{2}}\left(\tau_{j}-\mu_{j}\right)$ converges under the null to a normal with mean zero and variance $v_{j}^{2}$, where

$$
\begin{aligned}
& v_{1}^{2}=\operatorname{Var}_{H_{0}}\left\{\tau_{1}\right\}=2 \int \sigma^{4}(x) w^{2}(x) d x \int(\mathbf{K} * \mathbf{K})^{2}(x) d x, \\
& v_{2}^{2}=\operatorname{Var}_{H_{0}}\left\{\tau_{2}\right\}=2 \int \sigma^{4}(x) w^{2}(x) d x \int \mathbf{K}^{2}(x) d x \\
& \mu_{1}=E_{H_{0}}\left\{\tau_{1}\right\}=\frac{1}{n k^{d}} \int \sigma^{2}(x) w(x) d x \int \mathbf{K}^{2}(x) d x+o\left(\frac{1}{n k^{d}}\right), \\
& \mu_{2}=E_{H_{0}}\left\{\tau_{2}\right\}=\frac{1}{n k^{d}} \int \sigma^{2}(x) w(x) d x \mathbf{K}(0)+o\left(\frac{1}{n k^{d}}\right) .
\end{aligned}
$$

The next statistic was defined to avoid the calculation of high dimensional $\hat{m}$ :

$$
\tau_{3}=\frac{1}{n} \sum_{i=1}^{n}\left[\frac{1}{n} \sum_{j=1}^{n} \mathbf{K}_{k}\left(X_{i}-X_{j}\right) \hat{e}_{j}\right]^{2} w\left(X_{i}\right),
$$

where for ease of presentation and implementation $\mathbf{K}(\cdot)$ is the same kernel function as in the last subsection, and $k$ again its bandwidth. It is straightforward to derive from the above-mentioned paper that $n k^{\frac{d}{2}}\left(\tau_{3}-\mu_{3}\right)$ converges under the null to a normal variable with mean zero and variance $v_{3}^{2}$, where now

$$
\begin{aligned}
\mu_{3} & =E_{H_{0}}\left\{\tau_{3}\right\}=\frac{1}{n k^{d}} \int(\mathbf{K} * \mathbf{K})^{2}(x) d x \int \sigma^{2}(x) f^{2}(x) w(x) d x, \\
v_{3}^{2} & =\operatorname{Var}_{H_{0}}\left\{\tau_{3}\right\}=\int \sigma^{4}(x) f^{4}(x) w^{2}(x) .
\end{aligned}
$$

As the skedasticity functions $\sigma(x)$ are typically large where the density of $X$ is small, one obtains an efficient though natural weighting in the bias and variance term.

Finally, Guerre and Lavergne (2005) constructed a statistic which is particularly useful when applying their bandwidth choice method. It also avoids the estimation of $\hat{m}$, but requires the nonparametric estimation of the high dimensional densities of $X$. It is useful to introduce here test statistic $\tau_{4}$ as a function of bandwidth $k$ : 


$$
\begin{aligned}
\tau_{4}(k) & =\frac{1}{n-1} \sum_{i \neq j}\left\{Y_{i}-\hat{m}_{S}\left(X_{i}\right)\right\} \frac{\mathbf{K}_{k}\left(X_{i}-X_{j}\right)}{\sqrt{\hat{f}_{k}\left(X_{i}\right) \hat{f}_{k}\left(X_{j}\right)}}\left\{Y_{j}-\hat{m}_{S}\left(X_{j}\right)\right\} \\
& =\frac{1}{n-1} \sum_{i \neq j} \hat{e}_{i} w_{i j}(k) \hat{e}_{j},
\end{aligned}
$$

where $\hat{e}_{i}$ are the residuals under $H_{0}$, and $w_{i j}(k)$ the smoothing weights of the statistic. Later on, for the bandwidth choice procedure of Guerre and Lavergne (2005), we need the variances of $\tau_{4}$ and $\tau_{4}(k)-\tau_{4}\left(k_{P}\right)$, i.e., the difference of two statistics calculated for different bandwidths $k$ and $k_{P}$, respectively. To emphasize its dependency on $k$ and $k_{P}$, we give them here in terms of the smoothing weights $w_{i j}(k)$, i.e.,

$$
\begin{aligned}
v_{4}^{2}(k) & =\operatorname{Var}_{H_{0}}\left\{\tau_{4}(k)\right\}=2 \sum_{i, j} \sigma^{2}\left(x_{i}\right) w_{i j}^{2}(k) \sigma^{2}\left(x_{j}\right), \\
v_{4 b}^{2}\left(k, k_{P}\right) & =\operatorname{Var}_{H_{0}}\left\{\tau_{4}(k)-\tau_{4}\left(k_{P}\right)\right\} \\
& =2 \sum_{i, j} \sigma^{2}\left(x_{i}\right)\left\{w_{i j}(k)-w_{i j}\left(k_{P}\right)\right\}^{2} \sigma^{2}\left(x_{j}\right) d x .
\end{aligned}
$$

This will help us later on to understand better the idea of the procedure for selecting the testing bandwidth $k$.

All these tests have been proved to be consistent. We also studied other test statistics, for example, all those given in Dette et al. (2005), but have not considered them here further because they showed (even) less satisfactory performance.

\subsection{Calculating the critical value by resampling}

Asymptotics are of little help when calculating the critical value in practice since (1) bias and variance contain unknown expressions which have to be estimated nonparametrically, and (2) the convergence to them and the normality are quite slow. Therefore, it is common to use resampling (bootstrap or subsampling) methods to approximate the critical value for the particular sample statistic. The commonly used bootstrap procedure is

1. With bandwidth $h$, calculate the estimate $\hat{m}_{S}$ under the null hypothesis of additivity and its resulting residuals $\hat{e}_{i}, i=1, \ldots, n$.

2. With bandwidth $k$, calculate the estimator $\hat{m}$ (or the respective kernel expressions of the test statistic involving $k$ ) for the conditional expectation without the additivity restriction, and the corresponding residuals $\hat{u}_{i}, i=1, \ldots, n$.

3. With the results from step 1 and 2 , we can calculate our test statistics $\tau_{j}$.

4. Repeat step 1, but now with a bandwidth $h_{b}$ which depends on $h$ from step 1 . We call the outcome $\hat{m}_{S, h_{b}}$ and set $\epsilon_{i}=Y_{i}-\hat{m}_{S, h_{b}}\left(X_{i}\right)$ for $i=1, \ldots, n$. Draw random variables $e_{i}^{*}$ with $E\left[\left(e_{i}^{*}\right)^{l}\right]=\left(y_{i}-\hat{m}\left(x_{i}\right)\right)^{l}=: u_{i}^{l}$ (or $\hat{e}_{i}^{l}$, or even $\epsilon_{i}^{l}$, see discussion below) for $l=2,3$ (respectively $l=2$ only, see below again). Set $Y_{i}^{*}=\hat{m}_{S, h_{b}}\left(X_{i}\right)+e_{i}^{*}, i=1, \ldots, n$. Repeat this $B$ times. This defines $B$ different bootstrap samples $\left\{\left(X_{i}, Y_{i, b}^{*}\right)\right\}_{i=1}^{n}, b=1, \ldots, B$. 
5. For each bootstrap sample from step 4, calculate the test statistic $\tau_{j, b}^{*}, j=1,2,3,4$, $b=1, \ldots, B$. Then, for each statistic $\tau_{j}$, the critical value is approximated by the corresponding quantiles of the distribution of the $B$ bootstrap analogs: $F^{*}(v)=$ $\frac{1}{B} \sum_{b=1}^{B} \mathbb{1}\left\{\tau_{j, b}^{*} \leq v\right\}$. Note that they are generated under $H_{0}$.

This procedure is well known, has been proved to be consistent for many tests, and has therefore been applied, with slight modifications, to many non- or semiparametric testing problems. Unfortunately, it is not known how to choose the smoothing parameter $h_{b}$ in practice for the pre-estimation of the model that is used to generate the bootstrap samples. Note that in finite samples the correct choice will depend not only on $h$ but also on the choice of $k$, and the used residuals (see step 4.). Depending on the context, an interesting alternative would be to apply the smooth bootstrap, see Cao-Abad and González-Manteiga (1993) and Cao-Abad 1993. However, it 'suffers' from similar bandwidth choice problems.

A more and more popular alternative to bootstrapping is the subsampling procedure; see Politis et al. (1999). To date, as subsampling is commonly believed to converge slower than bootstrapping, it has been used almost exclusively when bootstrap fails; see Neumeyer and Sperlich (2006) for a purely nonparametric testing context. They also study the automatic choice of subsample size $N$ which turned out to work in their simulations. As this method can be remodeled to serve as a procedure for finding $h_{b}$, we introduce subsampling together with the automatic choice of the subsample size.

Let $\mathcal{Y}=\left\{\left(X_{i}, Y_{i}\right) \mid i=1, \ldots, n\right\}$ be the original sample and denote by $\tau \mathcal{Y}(k)$ the original statistic calculated from this sample, leaving aside the index $j=1,2,3,4$ to simplify notation. To determine the critical values, we need to approximate

$$
Q(z)=P\left(n \sqrt{k^{d}} \tau \mathcal{Y}(k) \leq z\right)
$$

Recall that under $H_{0}$ this distribution converges to a $N\left(\mu, v^{2}\right)$ with $\mu$ and $v$ given above. For finite sample size $n$, drawing $B$ subsamples $\mathcal{Y}_{b}$ - each of size $N$-we can approximate $Q$ under $H_{0}$ by

$$
\hat{Q}(z):=\frac{1}{B} \sum_{b=1}^{B} \mathbb{1}\left(N \sqrt{k_{N}^{d}} \tau \mathcal{Y}_{b}\left(k_{N}\right) \leq z\right) .
$$

Note that the awkward notation comes from the fact that we have to adjust all bandwidths to the new sample size $N$. For example, imagine $k=c_{0} \cdot n^{-\rho}$ for $c_{0}$ being a constant. Then, the subsample test $\tau\left(k_{N}\right)$ is calculated like $\tau$, but with bandwidth $k_{N}=c_{0} \cdot N^{-\rho}$. Certainly, under the alternative $H_{1}$, not only $n \sqrt{k^{d}} \tau \mathcal{Y}(k)$ but also $N \sqrt{k_{N}^{d}} \tau \mathcal{Y}_{b}\left(k_{N}\right)$ converges to infinity (while it does not so if $H_{0}$ is true). Then, demanding $N / n \rightarrow 0$ guarantees that $n \sqrt{k^{d}} \tau \mathcal{Y}(k)$ converges faster to infinity than the subsample analogs. Finally, $\hat{Q}$ underestimates the quantiles of $Q$ which yields the rejection of $H_{0}$ if it was false.

Actually, the optimal $N$ is a function of the nominal level $\alpha$. In order to find that $\alpha$ (or $N$ for a given $\alpha$ ), we test a null hypothesis of which we know that it is true, but 
suffers from the same smoothing bias as our original testing problem does. Such a null hypothesis is, for example, $H_{0}^{*}: m(x)-m_{S}(x)=\hat{m}(x)-\hat{m}_{S}(x)$ which is to be tested by an analogous statistic one uses to test the original problem $H_{0}: m(x)=m_{S}(x)$. For the desired level $\alpha$, apply that statistic to $H_{0}^{*}$ and use subsampling to determine its p-value. Now draw some pseudo sequences $\mathcal{Y}_{l}^{*}, l=1, \ldots, L$ from $\mathcal{Y}$ of size $n$ with the same distribution as $\mathcal{Y}$, and repeat this test $L$ times. From these repetitions, you can determine the empirical rejection level (estimated size) for the given $\alpha$. As you know that $H_{0}^{*}$ is true and therefore should be rejected exactly in $100 \alpha$ percent of all cases, you look for a subsample size $N$ producing this rejection level. In practice, you choose from a grid of potential $0<N<n$ the one whose rejection level comes the closest to $\alpha$ from below. For further details, see Politis et al. (1999), Delgado et al. (2001), or Neumeyer and Sperlich (2006). A drawback of this procedure is the computational cost.

\section{The choice of parameters}

The above-introduced statistics and procedures raise many questions of practical importance which typically remain open: bandwidth choice $h$ and $h_{-\delta}$ in step 1 , bandwidth choice $k$ in step 2, how to generate the bootstrap residuals $e_{i}^{*}$ in step 4 , how to choose $h_{b}$, and finally how many bootstrap samples are necessary to get a reasonable approximation of the distribution in step 5. We will discuss all these questions except the last one, giving mainly space to the discussions that are testing specifics.

Recall that the bandwidth choice problem in estimation is different from that in testing. Testing additivity is just an illustrative example here for a nonparametric testing problem with a semi- or nonparametric null hypothesis. We are generally interested in nonparametric specification tests where both the regression model and the testing problem could equally well have nothing to do with additivity, but for example with distribution assumptions, the link function, variable selection, heteroscedasticity, autocorrelation, endogeneity, jump detection, etc. The article is aimed to evoke a discussion about the resulting bandwidth choice problems and its impact on the test performance.

\subsection{The choice of bandwidths $h$}

The problem of finding an optimal $h$ is somewhat different from that of finding the optimal smoothing parameter $k$ which is directly linked to the optimal rate of the test statistic. In the latter case, it is clear that a theoretical optimal choice depends on the optimal rate at which the test can detect a deviation from the null hypothesis; see the next subsection. In most cases, the estimator of the null model can have faster convergence rates than that of the alternative, so the asymptotics of the test statistics provide no theoretical guideline for an optimal choice of $h\left(\right.$ or $h_{-\delta}$ ). To date, we have to rely on practical recommendations; but an optimal bandwidth choice for the null model in nonparametric testing is a potential topic for further investigation, cf. Rodriguez-Póo et al. (2012) and Sect. 4. 
As there exist data adaptive methods for finding the optimal bandwidth $k$ for the alternative (see next subsection), one could argue that $h$ should be chosen accordingly to $k$. This way, one would guarantee that the same smoothness is imposed on the regression function regardless of whether it is estimated under the null hypothesis or not. However, it is not clear whether this is always wanted. Moreover, we will see that the adaptive choice of $k$ is computationally intensive and that also $h_{b}$ (cf. Sect. 2.3) depends on $h$. So each bandwidth selection would depend on each other, and it is unclear where to start. Apart from the fact that we could not find any suggestion in the literature pointing in this direction, this would lead to a computationally quite complex procedure. Intuitively, it seems to be desirable to look for a reasonable estimation of the null model. This is only guaranteed with a reasonable bandwidth choice of $h$ beforehand. All in all, given the state of the art one would - and typically doesrecommend to follow this intuitive argument and use (generalized) cross-validation (cv henceforth) or plug-in methods; see Vieu (1993) for a classic survey, and Köhler et al. (2013) for a recent review on bandwidth selection methods in kernel regression.

For internalized marginal integration estimators, no explicit bandwidth selection procedure is available. Here, we follow the cross-validation idea. Recall that in practice it is often preferred to simply divide all explanatory variables by its standard deviation and then use the same bandwidth for all directions. On the one hand, this is certainly suboptimal from a strictly statistical point of view, but on the other hand this follows the idea of comparable smoothness in all directions. In such a case, we have only two parameters to choose: $h$ and $h_{-\delta}$. This can be done by minimizing the cv criterion

$$
C V\left(h, h_{-\delta}\right)=\frac{1}{n} \sum_{i=1}^{n}\left\{Y_{i}-\hat{m}_{S}^{-}\left(X_{i}\right)\right\}^{2}
$$

where $\hat{m}_{S}^{-}\left(X_{j}\right)=\hat{\psi}+\sum_{\delta=1}^{d} \hat{m}_{\delta}^{-}\left(X_{j \delta}\right)$ with

$$
\hat{m}_{\delta}^{-}\left(X_{j \delta}\right)=\frac{1}{n-1} \sum_{i \neq j}^{n} Y_{i} K_{h}\left(X_{j \delta}-X_{i \delta}\right) \frac{\hat{f}_{-\delta}\left(X_{i,-\delta}\right)}{\hat{f}\left(X_{i \delta}, X_{i,-\delta}\right)} .
$$

As explained in Kim et al. (1999), the fraction of estimated densities is a weighting necessary to avoid an omitted-variable bias. The leave-out or not leave-out of $X_{j}$ has a minor impact in that fraction, so that the definition of the cv criterion via (9) and (10) constitutes an acceptable simplification of the classical cross-validation, like it has already been proposed by Nielsen and Sperlich (2005) in the context of smooth backfitting. So we minimize here the average mean squared error for the null model. This does not necessarily give the bandwidths to minimize the estimation errors of the individual additive components; but, recall that all test statistics look for the prediction (or fitting) power of the total regression.

Further discussion of potential bandwidth selectors for this estimator will not help to deepen the understanding of the considered testing problem. However, since we already reviewed alternative estimators for nonparametric additive models, we should conclude here giving a brief review for choosing the corresponding smoothing 
parameters. Hastie and Tibshirani (1990) proposed cross-validation and generalized cv criteria. Linton and Nielsen (1995) and Severance-Lossin and Sperlich (1999) proposed different plug-in estimators for the original marginal integration. As said, for its internalized version, we are not aware of any specific proposal. Mammen and Park (2005) as well as Nielsen and Sperlich (2005) introduced several plug-in and cv methods for the smooth backfitting estimator. For the classical backfitting with splines, often the so-called generalized cv is recommended. A more detailed discussion, though for the Bayesian approach, can be found in Fahrmeir et al. (2010). We stop here also because for each specific null hypothesis one has a specific bandwidth choice problem; recall that testing for additivity is only an example here.

\subsection{The choice of bandwidths $k$}

It is known that a bandwidth $k$ which is optimal for estimation is usually suboptimal for testing. More specifically, the optimal smoothing parameter for testing has faster convergence rates; we actually should undersmooth. As cv bandwidths have a tendency to undersmooth in practice, they are quite popular in nonparametric testing.

As an alternative, let us consider the adaptive testing approach introduced in Spokoiny (1998). The (where necessary modified and adapted) method is the same for each of our four test statistics, so that we skip the index $j$ of $\tau_{j}, j=1,2,3,4$ for a moment, but use the notation $\tau(k)$ indicating the testing bandwidth applied. Adapted to our problem, it works as follows. Consider simultaneously a family of tests $\{\tau(k), k \in \mathfrak{K}\}$, where $\mathfrak{K}=\left\{k_{1}, k_{2}, \ldots, k_{P}\right\}$ is a finite set of reasonable bandwidths. The theoretical maximal number $P$ depends on $n$; for the sequences $\left\{k_{j}\right\}_{j=1}^{P}$, see the particular paper.

Horowitz and Spokoiny (2001) proposed to look at

$$
\tau^{\max }=\max _{k \in \mathfrak{K}} \frac{\tau(k)-E_{H_{0}}[\tau(k)]}{\operatorname{Var}^{1 / 2}[\tau(k)]} \text {, where }
$$

$E_{H_{0}}[\cdot]$ indicates the expectation under $H_{0}$, and the variance has to be estimated with an estimator of $\sigma^{2}$ that is consistent under $H_{1}$. This studentizing under the null is to correct for the deviations in distribution caused by the different bandwidths $k$. Instead of $\operatorname{Var}^{1 / 2}[\tau(k)]$, one could take something proportional to it without losing consistency (though asymptotic efficiency) as long as it corrects for the standard deviations caused by the different $k \in\left\{k_{1}, \ldots, k_{P}\right\}$. We will call this method $H S$ when explicitly estimating the required moments, and $k_{o}$ will denote the bandwidth giving $\tau^{\text {max }}$.

Guerre and Lavergne (2005) suggested a procedure to select $k$ for $\tau_{4}$ (in a simpler testing context) of which they stated it would select a bandwidth even more tailored than the HS one for testing. They presupposed to dispose of centered (under $H_{0}$ ) test statistics - therefore we will take again $\left\{\tau_{4}(k)-E_{H_{0}}\left[\tau_{4}(k)\right]\right\}$. Instead of dividing by its standard deviation, they selected $k$ along the criterion

$$
k_{o}=\arg \max _{k \in \mathfrak{K}}\left\{\tau_{4}(k)-\widehat{E}_{H_{0}}\left[\tau_{4}(k)\right]-\kappa_{n} \hat{v}_{4 b}\left(k, k_{P}\right)\right\}, \kappa_{n}=2 \sqrt{2 \ln P} .
$$


In our context, $k_{P}$ is the largest bandwidth in $\mathfrak{K}$. Their final test statistic was $\tau_{4}\left(k_{o}\right) / v_{4}\left(k_{P}\right)$. Let us denote this method by $G L$ in the following.

A quite different approach was proposed by Gao and Gijbels (2008). For testing a fully parametric null hypothesis, they considered a simplified version of $\tau_{4}$ and calculated the Edgeworth expansion of its size and power function. Then, for the obtained expressions, they chose bandwidth $k$ such that it maximized the power while holding the size. These expansions and its bootstrap approximates are subject to serious changes when the null hypothesis becomes non- or semiparametric. Although this has not yet been analyzed in detail, it is clear that the approximation will not only change importantly but also be much less reliable, e.g., concerning the distribution. Especially the size function-as it depends on the (estimated) null model—will be affected by the smoothing and bootstrap bias. Summarizing, it is unclear how the semiparametric analog to this solution would look like, and it is unlikely that this approximation would work well until the samples size is huge.

In contrast, it is obvious how to extend the method of Horowitz and Spokoiny (2001) or the one of Guerre and Lavergne (2005) to problems with semi- or nonparametric null hypotheses, see Rodriguez-Póo et al. (2012). A particularity of the bootstrap analogs of $\tau^{\max }=\tau\left(k_{o}\right)$ is that one first needs to calculate the bootstrap statistics $\tau_{b}^{*}(k)$ for all $k \in \mathfrak{K}$ to get $\left(\tau^{\max }\right)_{b}^{*}$ as this is not necessarily equal to $\tau_{b}^{*}\left(k_{o}\right)$ when $k_{o}$ refers to the bandwidth that maximizes (only) the original statistic. In other words, for each bootstrap sample, this bandwidth can be different. In fact, the bootstrap tries to simulate an extreme value distribution. The empirical moments of the bootstrap statistics $\tau_{b}^{*}(k)$ can be used to estimate $E_{H_{0}}[\tau(k)]$, respectively, $\operatorname{Var}^{1 / 2}[\tau(k)]$, in practice. This is what we will try and study in our simulations for $\tau^{\max }$ in (11), called henceforth HSB as it refers to the purely bootstrap-based HS version. The direct estimation of these expectations and variances is often quite hard if not infeasible.

\subsection{The choice of bootstrap residuals}

From a theoretical point of view, wild bootstrap errors should be drawn from the residuals of the alternative model, i.e., $u_{i}$ should be used in Section 2.3, step 4. Clearly, this would maximize the power, as the variance of $\epsilon_{i}$ (or $\hat{e}_{i}$ ) can increase a lot with increasing distance between $H_{0}$ and the true model. For consistency, it is sufficient to have this variance bounded, but it is evident that these phenomena deteriorate the power of the test. In the study of Dette et al. (2005), the power loss was negligible when using residuals from the null instead of taking residuals from the alternative.

Arguments in favor of using $\epsilon_{i}$ exist only under other, mainly practical aspects: often the size distortion in bootstrap tests is worse when using $u_{i}$ or $\hat{e}_{i}$. Moreover, when using adaptive procedures (i.e., the statistic is evaluated over a range of testing bandwidths $k$ ) as described in Subsection 3.2, it is not that clear which of the $u_{i}(k)$ to use or whether the $u_{i}$ should be estimated independently of the $k$-choice for the test. So the size argument seems to be decisive though we admit that, if no adaptive choice of $k$ is made, it would be desirable to use $u_{i}$ as long as one can control for the size distortion.

The second question is what kind of distribution should be used for generating the random errors. In step 4 of the bootstrap procedure described in Subsection 2.3, a 
distribution is commonly taken that gives $e_{i}^{*}$ with $E\left[\left(e_{i}^{*}\right)^{l}\right]=\epsilon_{i}^{l}$ for $l=2$ up to 3 (or even more). The so-called golden-cut wild bootstrap is quite popular, see, e.g., Härdle and Mammen (1993). More recently, in the context of size distortion of bootstrap tests, Davidson and Flachaire (2008) argued that for problems with moderate sample size, the disadvantages of the higher-order moment adapting bootstraps outweigh their asymptotic advantages. To check this, we compare different methods in our simulations.

\subsection{The choice of bootstrap bandwidth $h_{b}$}

In general, for many test statistics, one knows that the mean of $\hat{m}_{h}(x)-m(x)$ under the conditional distribution of $Y_{1}, \ldots, Y_{n} \mid X_{1}, \ldots, X_{n}$, respectively of $\hat{m}_{h}^{*}(x)-\hat{m}_{h_{b}}(x)$ under the conditional distribution of $Y_{1}^{*}, \ldots, Y_{n}^{*} \mid X_{1}, \ldots, X_{n}$, can be approximated by

$$
\begin{aligned}
& E_{Y \mid X}\left(\hat{m}_{h}(x)-m(x)\right) \approx h^{2} \frac{\mu(K)}{2} m^{\prime \prime}(x), \\
& E^{*}\left(\hat{m}_{h}^{*}(x)-\hat{m}_{h_{b}}(x)\right) \approx h^{2} \frac{\mu(K)}{2} \hat{m}_{h_{b}}^{\prime \prime}(x),
\end{aligned}
$$

where $\mu(K)=\int u^{2} K(u) d u$, see Rosenblatt (1969). Then, to guarantee that (13) is well approximated by the bootstrap version (14), we need that $\left\{\hat{m}_{h_{b}}^{\prime \prime}(x)-m^{\prime \prime}(x)\right\} \rightarrow 0$. The optimal bandwidth $h_{b}$ for estimating the second derivative is known to be much larger (in rates) than the optimal $h$ for estimating the function itself. We can even give the optimal rate. For example, the optimal rate to estimate $m_{S}^{\prime \prime}$ is of the order $n^{-1 / 9}$ (instead of $n^{-1 / 5}$ ) in the one-dimensional case. This gives the optimal bootstrap bandwidth to construct uniform confidence bands, see Härdle and Marron (1991) and Cao-Abad and González-Manteiga (1993); compare also with González-Manteiga et al. (2004) for the $d>1$-dimensional case. They give an explicit plug-in formula for the optimal pre-smoothing bandwidth, but in a different context and containing many unknown expressions. In our simulation study, we will come back to this optimal rate for $h_{b}$. However, it will also be seen that the typical comment $h_{b}$ has to be oversmoothing compared to $h$ is not very helpful; neither taking any $h_{b}>h$ nor setting $h_{b}=h \cdot n^{\frac{1}{5}-\frac{1}{9}}$ will guarantee a size $\approx \alpha$ with non-trivial power. In fact, the proper choice of $h_{b}$ has even not been solved appropriately if we just use it for constructing confidence bands in estimation, see Neumann and Polzehl (1998) or Claeskens and Keilegom (2003). Typically, it is recommended to undersmooth $h$ or interpret the band as a band around the estimator, not the function. Then one could set $h=h_{b}$, at least in the univariate case $d=1$. In testing, this choice problem is unfortunately more involved. Our simulations show very well that one has a serious size problem due to the smoothing bias in the bootstrap samples. There exist econometric papers that do simulations under $H_{0}$ with large bandwidths, and under $H_{1}$ with small bandwidthsreplicating their simulations, one can see that the large bandwidths cause trivial power under $H_{1}$ and the small bandwidths reject under $H_{0}$ much too often.

The practitioner needs a clear guideline on how to choose $h_{b}$ which we unfortunately cannot find in the literature. A proper and detailed development of such a selection 
strategy is beyond the scope of this paper; we rather intend to review and highlight the existing but commonly ignored problems in nonparametric specification testing in econometrics. Only in the note of Barrientos and Sperlich (2010) it was suggested to apply the same idea used for the automatic choice of a proper subsample size $N$ in subsampling (cf. Sect. 2.3), but has so far not really been studied. This will be tried and analyzed in our simulation study as it is the only hint we found in the literature.

More specifically: imagine $h_{b}$ as a function of $\alpha$, and for simplification fix also $k$. The procedure can be extended to GL-, HS-, and HSB-data adaptive choices of $k$.

1. For a given nominal level $\alpha$ consider the testing problem $H_{0}^{*}: m(x)-m_{S}(x)=$ $\hat{m}(x)-\hat{m}_{S}(x)$ which will be tested with an analogous statistic one wants to use for the original problem $H_{0}: m(x)=m_{S}(x)$.

2. Draw some pseudo sequences $\mathcal{Y}_{l}^{*}, l=1, \ldots, L \geq 100$ from $\mathcal{Y}$ of size $n$ with the same distribution as $\mathcal{Y}$.

3. Take $h_{b}$ from a bandwidth grid and do a bootstrap test to check $H_{0}^{*}$ for all the $L$ samples $\mathcal{Y}_{l}^{*}$ generated above.

4. Let $\alpha^{*}$ be the percentage of rejections of $H_{0}^{*}$ in the $L$ samples. If $\alpha^{*}=\alpha$, then you have found the appropriate $h_{b}$, else go back to step 3 to try with a different $h_{b}$.

5. In practice, none of the $h_{b}$ of the grid will produce $\alpha^{*}=\alpha$ exactly. Then take for the original test the bandwidth $h_{b}$ which minimizes $\left(\alpha-\alpha^{*}\right)$ for $\alpha^{*} \leq \alpha$.

\section{Simulation results}

To study all the points listed in the last section, we performed a comprehensive simulation study. We give here only a summary, for example, limiting the presentation to $w(x) \equiv 1$ for $\tau_{j}, j=1,2,3,4$, one particular model, one specific (random) design, and sample size $n$ to 150 . We draw three-dimensional i.i.d. explanatory variables

$$
X_{i} \sim N\left(0, \Sigma_{X}\right) \text { with } \Sigma_{X}=\left(\begin{array}{ccc}
1 & 0.2 & 0.4 \\
0.2 & 1 & 0.6 \\
0.4 & 0.6 & 1
\end{array}\right)
$$

and i.i.d. error terms $e_{i} \sim N\left(0, \sigma_{e}^{2}\right)$ to obtain

$$
Y_{i}=X_{1, i}+X_{2, i}^{2}+2 \sin \left(\pi X_{3, i}\right)+a X_{2, i} X_{3, i}+e_{i}, \quad i=1, \ldots, n
$$

with $a=0$ to generate an additive separable model, and $a=2$ for the alternative. The null hypothesis is additivity. Unless otherwise indicated, $\sigma_{e}=1$. For the unrealistic situation where $\Sigma_{X}$ is the identity matrix (i.e., with covariates being independent from each other), the problem is greatly simplified. A much stronger correlated design than ours leads already to identification problems for moderate sample sizes.

All results in the tables are calculated from 1,000 replications using 250 bootstrap samples or subsamples. For real data applications, 250 bootstrap samples are certainly very few; but in our simulations, the results differed little when we increased the number to 500. We used the multiplicative quartic kernel throughout. 
When we used the weighting function $w(\cdot)$ for trimming to get rid of the boundary effects, we certainly got somewhat different numerical results. The relative findingsmay it be size problems or the ranking of the tests by power-did not change. Therefore, we present here only results without trimming or any other additional weighting.

We start with the bandwidth choice for estimating the null model. Let the parameter responsible for the size, $h_{b}$, depend on $\alpha$ (the level), $k$, and $h$, but not vice verse. Then it is no problem when $h$ is chosen by cv in each simulation run as proposed in Sect. 3.1. For the nuisance directions $X_{-\delta}$, see Eq. (10), it is known that a much larger bandwidth $h_{-\delta}$ can (cf. Hengartner and Sperlich (2005)) and has to be used (cf. Dette et al. 2005), always supposing that these covariates have smooth densities. We tried therefore with setting $h_{-\delta}=c \cdot h$ where $c \in\{3,4,5,6,7,8\}$, based on the recommendations of the above quoted papers. The optimum seems to be between 5 and 6 when choosing $h_{-\delta}$. But now recall our discussion about the fact that minimizing the average mean squared error for estimation might not be the optimal choice for optimizing the test performance. That already the smoothing parameter choice for the null model can have an important impact on the test performance will easily be seen when in the following we always contrast the choices $h_{-\delta}=5 \cdot h$ with $h_{-\delta}=6 \cdot h$.

We also tried different bootstrap residuals (cf. Sect. 2.3). Our simulations mainly seem to confirm the arguments discussed above: The power loss caused by not using residuals from the alternative was negligible; so the size problem was decisive for our final decision. Below we report only results referring to $e_{i}^{*}=\varepsilon_{i} \cdot \epsilon_{i}$, where the $\varepsilon_{i}$ are i.i.d. $N(0,1)$. When they were drawn from the golden-cut distribution

$$
\varepsilon_{i}=\left\{\begin{array}{lll}
-(\sqrt{5}+1) / 2 & \text { with probability } & p=(\sqrt{5}+1) /(2 \sqrt{5}) \\
(\sqrt{5}+1) / 2 & \text { with probability } & 1-p
\end{array},\right.
$$

all our results became much worse (less precise sizes and less power) which is in accordance with Davidson and Flachaire (2008). However, we admit that it might be interesting to study anew the effect of which residuals to take (i.e., $u_{i}, \hat{e}_{i}$ or $\epsilon_{i}$ ) when trying different choice procedures for $h_{b}$.

For the bandwidth of the test, recall Section 3.2, we let $k$ run over a grid of $P=10$ bandwidths from $k_{\max }=k_{P}=6$, the range of the support of each covariate, to $k_{\text {min }}=k_{1}=3 / n^{1 / d}$, such that the approximate $\operatorname{support}(X) \approx[-3,3]^{d}$ can be covered by $n$ cubes of volume $\left(2 k_{\text {min }}\right)^{d}$, which is the support of each multiplicative quartic kernel. For $\tau_{j}, j=1, \ldots, 4$ we first study the results for all $k$ and compare them with $\tau_{j}^{\max }(\mathrm{HSB}), j=1, \ldots, 4$. We verified that the $\tau_{j}^{\max }$ did not take values at the boundaries $k_{\min }$ or $k_{\max }$. We speak of "adaptive" tests when referring to the $\tau_{j}^{\max } \mathrm{HS}$ or HSB method, or the automatic choice of $k$ along GL. In these cases, we call the chosen bandwidth $k_{o}$. Non-adaptive procedures certainly vary over the range from $k_{1}$ to $k_{P}$.

Another challenging point is the choice of $h_{b}$. We first give results obtained for particular $h_{b}$ and let $k$ run. In a second step, we do it vice versa. To choose $h_{b}$ as a function of $h$ and validate the "oversmoothing" argument including the optimal rate $n^{-1 / 9}$ (recall discussion and justification in Sect. 3.4) we set

$$
h_{b}=h \cdot n^{1 / 5-1 / \gamma} \text {, for } \gamma \in\{4,5,6,7,8,9,10\} \text {. }
$$



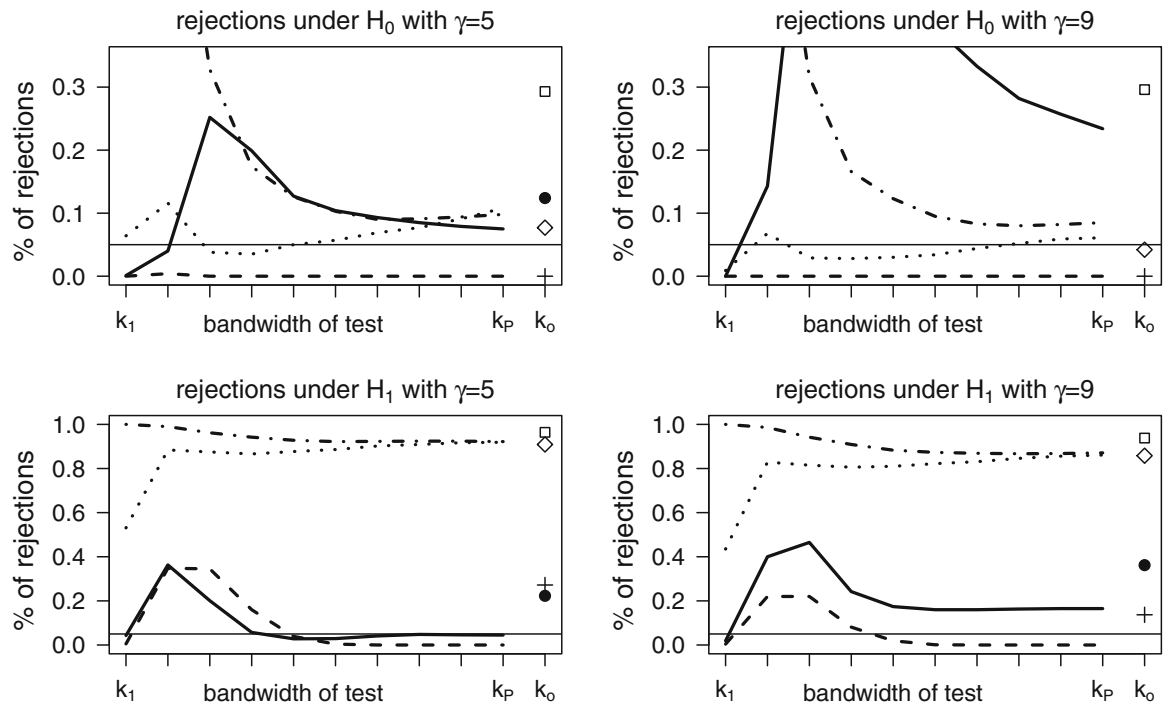

Fig. 1 When $h_{-\delta}=5 h$ : Real sizes (upper line) and rejection levels (lower line) over $k$, where $k_{o}$ stands for adaptive choice referring to the bootstrap studentized (HSB) $\tau_{j}^{\text {max }}$. Nominal size was $\alpha=5 \%$ (thin line). $\tau_{1}:$ solid line; $\tau_{1}^{\max }:$ bullet (out of range in the upper right graph); $\tau_{2}:$ dashed line; $\tau_{2}^{\max }:$ plus; $\tau_{3}$ : dotted line; $\tau_{3}^{\max }:$ diamond; $\tau_{4}:$ dots and dashes; $\tau_{4}^{\max }:$ square.

Supposing that our cv bandwidth follows $h \propto n^{-1 / 5}$ one starts with an $h_{b}<h$ for $\gamma=4$ and goes up to $h_{b} \propto n^{-1 / 10}$ including the optimal rate $n^{-1 / 9}$. As $h_{b}$ is chosen data adaptively and thus changes for each sample, we report always the pre-fixed $\gamma$.

Clearly, since we calculated simulation results for all combinations of bandwidths, tests statistics, selection, and resampling methods, its presentation would generate a serious data and information overflow. So we have decided to condense the presentation of numerical results in the following way. First, we concentrate on one bootstrap method as described above. Second, we only present the rejection levels for the nominal size of $\alpha=5 \%$ (error of the first kind) and some p-values. Third, recall the further settings mentioned at the beginning of this section.

Figures 1 (for $h_{-\delta}=5 h$ ) and 2 (for $h_{-\delta}=6 h$ ) show the real size and rejection levels for nominal size $\alpha=5 \%$ over different test bandwidths $k$ for given $\gamma=5$ and 9 respectively, i.e., fixing the rate of (over-)smoothing for the pre-estimation to later generate the bootstrap samples. The results corresponding to $k_{o}$ refer to the adaptive tests $\tau_{j}^{\max }(H S B)$, cf. Sect. 3.2. It can be seen how power and size problems vary over the range of test bandwidth $k$. At least for the presented $\gamma=5$ and 9 tests $\tau_{4}$ and $\tau_{1}$ have serious size problems, $\tau_{2}$ is much too conservative, and even for $\tau_{3}$ the proper choice of $k$ seems to be crucial. Finally, $\tau_{1}$ and $\tau_{2}$ exhibit quite poor power. The impact of the bandwidth for estimating the null hypothesis is also interesting, which in our case is only identified via the variation of $h_{-\delta}$. All graphs in Figure 2 have basically the same shape like in Fig. 1, but on a different scale. Similar statements hold for the versions with HSB adaptive choices of $k$. Note that we tried some more test statistics, but found all others to perform worse. 

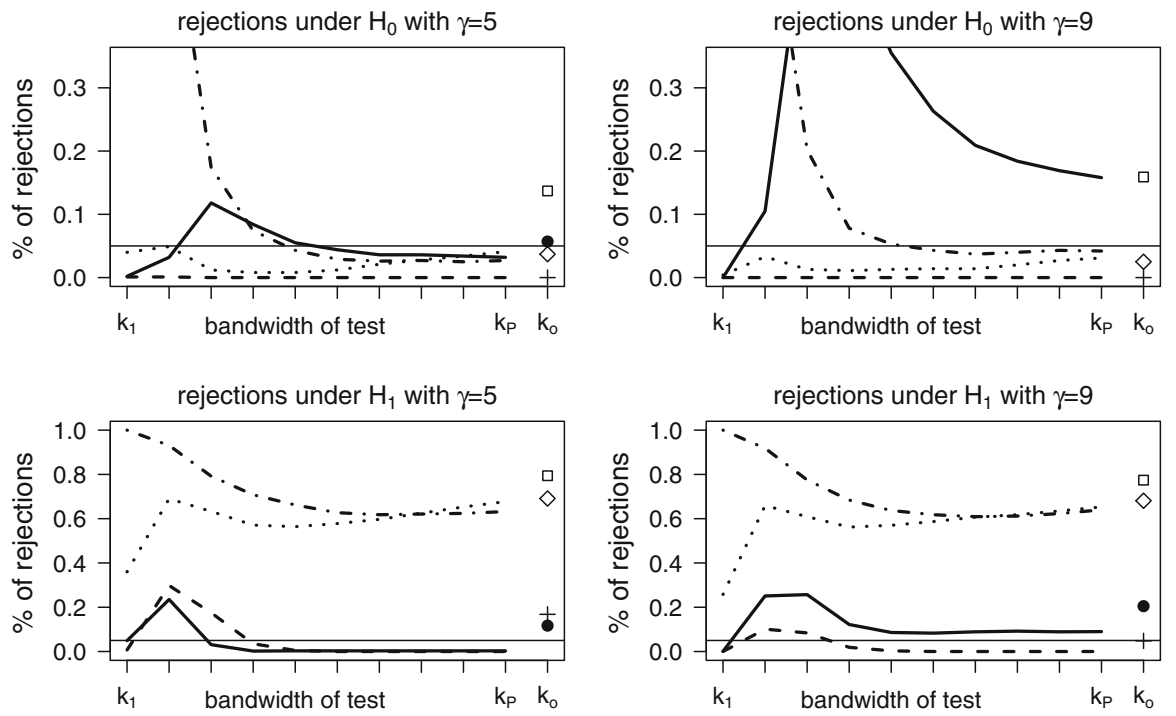

Fig. 2 When $h_{-\delta}=6 h$ : Real sizes (upper line) and rejection levels (lower line) over $k$, where $k_{o}$ stands for adaptive choice referring to the bootstrap studentized (HSB) $\tau_{j}^{\text {max }}$. Nominal size was $\alpha=5 \%$ (thin line). $\tau_{1}:$ solid line; $\tau_{1}^{\max }:$ bullet (out of range in the upper right graph); $\tau_{2}:$ dashed line; $\tau_{2}^{\max }:$ plus; $\tau_{3}$ : dotted line; $\tau_{3}^{\max }:$ diamond; $\tau_{4}:$ dots and dashes; $\tau_{4}^{\max }:$ square.

Next, in Figs. 3 (for $h_{-\delta}=5 h$ ) and 4 (for $h_{-\delta}=6 h$ ) are given the proportions of rejections for a nominal level $\alpha=5 \%$ under $H_{0}$ and $H_{1}$ over the range of $\gamma=4$ to $\gamma=9$. We first set $k$ equal to $k_{6}:=k_{1}+5\left(k_{P}-k_{1}\right) /(P-1)$, then repeated the comparison with the HSB adaptive choice $k_{o}$, i.e., for $\tau_{j}^{\max }(H S B), j=1, \ldots, 4$. These results basically show that the problem is not simply solved by a different smoothing in the pre-estimation. Oversmoothing, as generally recommended from a theoretical point of view, seems even to go into the wrong direction for some statistics. In particular, the hope that the intuition [see Eqs. (13) and (14)] might give us a hint or even provide a rule of thumb for the choice of $h_{b}$ is not confirmed. Again, $\tau_{3}$ outperforms the other tests having almost as much power as $\tau_{4}$, but with mitigated size problems. Test $\tau_{2}$ is the only one that holds throughout the level, but shows only some power in its k-adaptive version (right hand side, i.e., with $k_{o}$ ).

In Sect. 3.2, we discussed several strategies to find a data-driven bandwidth $k_{o}$ tailored toward the test. However, recall that both methods HS and GL are not always easy to implement; the GL requires for example good estimates of the bias $\mu_{4}$ and of the variances $v_{4}^{2}(k)$ and $v_{4 b}^{2}\left(k, k_{P}\right)$. In fact, estimates of the conditional variance of $Y$ are necessary which are also consistent under the alternative, and in the respective papers estimators were proposed which are only reasonable for one- or two-dimensional problems. Moreover, it is well known that the asymptotic expressions are little helpful for moderate samples. This is why we suggested to use the bootstrap to approximate also the required moments and called that approach HSB when applied to $\tau^{\max }$ in (11). For GL, one has also to estimate the correlation of test statistics corresponding to different bandwidths which can be quite tedious even when using bootstrap. We therefore limit 

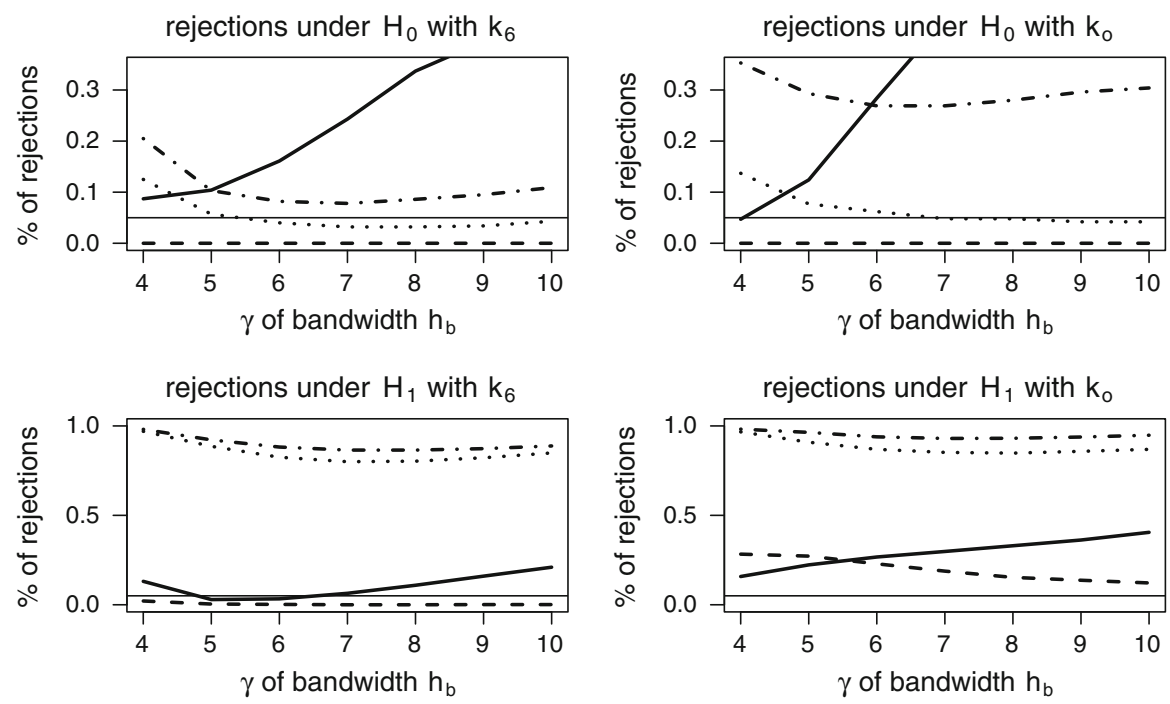

Fig. 3 When $h_{-\delta}=5 h$ : Real sizes (upper line) and rejection levels (lower line) over $\gamma\left(\gamma_{1}=4\right.$ to $\gamma_{7}=10$ ) for $k_{6}$ and adaptive bandwidth (HSB) $k_{o}$. Nominal size was $\alpha=5 \%$. $\tau_{1}$ : solid line; $\tau_{2}:$ dashed line; $\tau_{3}$ : dotted line; $\tau_{4}:$ dots and dashes
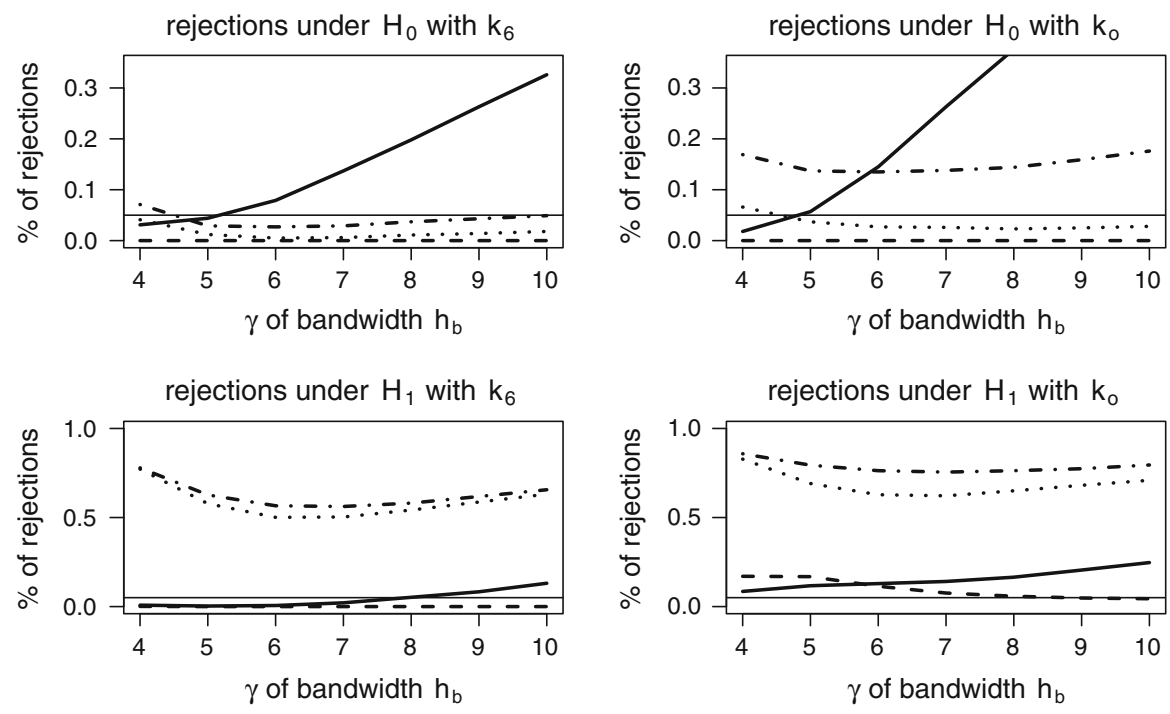

Fig. 4 When $h_{-\delta}=6 h$ : Real sizes (upper line) and rejection levels (lower line) over $\gamma\left(\gamma_{1}=4\right.$ to $\left.\gamma_{7}=10\right)$ for $k_{6}$ and adaptive bandwidth (HSB) $k_{o}$. Nominal size was $\alpha=5 \% . \tau_{1}:$ solid line; $\tau_{2}:$ dashed line; $\tau_{3}$ : dotted line; $\tau_{4}:$ dots and dashes

here our simulation comparison to the following: We estimated explicitly the required moments of $\tau_{4}$ for the HS and GL procedure, i.e., the statistic for which the GL method has been derived with explicit estimators. Also the HS was developed for a modified version of $\tau_{4}$ together with explicit expressions for the required moment estimators. 

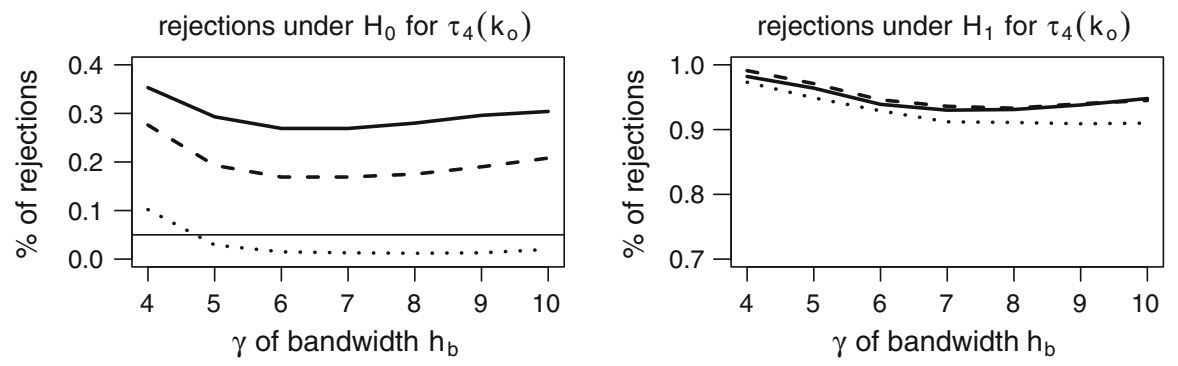

Fig. 5 When $h_{-\delta}=5 h$ : Real sizes (left) and rejection levels (right) of $\tau_{4}$ over $\gamma\left(\gamma_{1}=4, \gamma_{7}=10\right)$ with different k-adaptive versions: HSB: solid line; HS: dashed line; GL: dotted line. Nominal size was $\alpha=5 \%$
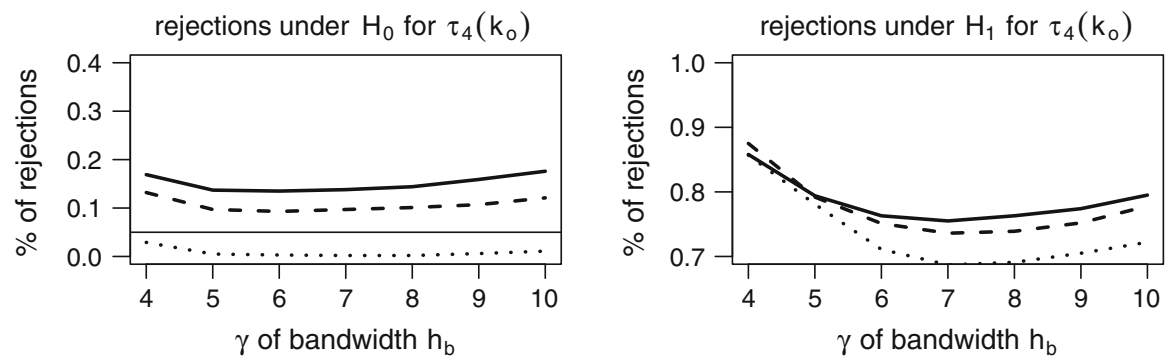

Fig. 6 When $h_{-\delta}=6 h$ : Real sizes (left) and rejection levels (right) of $\tau_{4}$ over $\gamma\left(\gamma_{1}=4, \gamma_{7}=10\right)$ with different k-adaptive versions: HSB: solid line; HS: dashed line; GL: dotted line. Nominal size was $\alpha=5 \%$

Table $1 P$-values of $k$-adaptive tests under $H_{0}$ and $H_{1}$ when $h_{-\delta}=5 h$

\begin{tabular}{|c|c|c|c|c|c|c|c|c|c|c|}
\hline \multirow[b]{2}{*}{$\gamma=$} & \multicolumn{5}{|c|}{ under $H_{0} \quad a=0.0$} & \multicolumn{5}{|c|}{ under $H_{1} \quad a=2.0$} \\
\hline & 5 & 6 & 7 & 8 & 9 & 5 & 6 & 7 & 8 & 9 \\
\hline$\tau_{4}\left(k_{0}-G L\right)$ & 0.485 & 0.558 & 0.577 & 0.567 & 0.544 & 0.014 & 0.019 & 0.022 & 0.022 & 0.022 \\
\hline$\tau_{4}^{\max }(H S)$ & 0.269 & 0.304 & 0.309 & 0.299 & 0.285 & 0.009 & 0.014 & 0.017 & 0.017 & 0.016 \\
\hline$\tau_{4}^{\max }(H S B)$ & 0.243 & 0.275 & 0.278 & 0.269 & 0.254 & 0.009 & 0.013 & 0.016 & 0.016 & 0.016 \\
\hline$\tau_{1}^{\max }(H S B)$ & 0.350 & 0.270 & 0.199 & 0.151 & 0.120 & 0.191 & 0.191 & 0.193 & 0.189 & 0.180 \\
\hline$\tau_{2}^{\max }(H S B)$ & 0.663 & 0.746 & 0.828 & 0.880 & 0.912 & 0.196 & 0.220 & 0.256 & 0.289 & 0.315 \\
\hline$\tau_{3}^{\max }(H S B)$ & 0.350 & 0.425 & 0.476 & 0.503 & 0.515 & 0.016 & 0.025 & 0.030 & 0.032 & 0.030 \\
\hline
\end{tabular}

For comparison, we present the HS and GL together with our HSB approach which we implemented for all tests. Figures 5 (for $h_{-\delta}=5 h$ ) and 6 (for $h_{-\delta}=6 h$ ) give the percentages of rejection over the range of $\gamma$ s considered in this study for all these k-adaptive methods. Only the GL method provides a test that holds the level for this model and sample size. As the other procedures produce much too liberal tests, it is not surprising that their power seems to be stronger.

Certainly, the 5\% rejection level is just a particular size. Holding this $\alpha$ level does not mean that the procedure indeed fits well the (true) distribution of the test under the null and therefore would work well in general. Tables 1 and 2 provide the p-values for 
Table $2 P$-values of $k$-adaptive tests under $H_{0}$ and $H_{1}$ when $h_{-\delta}=6 h$

\begin{tabular}{|c|c|c|c|c|c|c|c|c|c|c|}
\hline \multirow[b]{2}{*}{$\gamma=$} & \multicolumn{5}{|c|}{ under $H_{0} \quad a=0.0$} & \multicolumn{5}{|c|}{ under $H_{1} \quad a=2.0$} \\
\hline & 5 & 6 & 7 & 8 & 9 & 5 & 6 & 7 & 8 & 9 \\
\hline$\tau_{4}\left(k_{o}-G L\right)$ & 0.712 & 0.773 & 0.779 & 0.761 & 0.732 & 0.095 & 0.116 & 0.123 & 0.121 & 0.116 \\
\hline$\tau_{4}^{\max }(H S)$ & 0.495 & 0.527 & 0.521 & 0.499 & 0.472 & 0.080 & 0.103 & 0.107 & 0.101 & 0.091 \\
\hline$\tau_{4}^{\max }(H S B)$ & 0.482 & 0.514 & 0.507 & 0.484 & 0.456 & 0.079 & 0.100 & 0.104 & 0.097 & 0.088 \\
\hline$\tau_{1}^{\max }(H S B)$ & 0.548 & 0.464 & 0.364 & 0.285 & 0.231 & 0.348 & 0.383 & 0.398 & 0.391 & 0.369 \\
\hline$\tau_{2}^{\max }(H S B)$ & 0.756 & 0.839 & 0.901 & 0.938 & 0.958 & 0.328 & 0.397 & 0.457 & 0.500 & 0.527 \\
\hline$\tau_{3}^{\max }(H S B)$ & 0.520 & 0.600 & 0.639 & 0.655 & 0.655 & 0.070 & 0.102 & 0.108 & 0.099 & 0.088 \\
\hline
\end{tabular}

all adaptive tests we have studied so far. We let $\gamma$ of $h_{b}$ run from 5 to 9 . One can see that only $\tau_{3}^{\max }(H S B)$ and $\tau_{4}\left(k_{o}-G L\right)$ can compete, giving p-values of about 0.5 or more under $H_{0}$ and reasonable power (i.e., small $P$-values) under the alternative $H_{1}$.

Next we turn to the automatic choice of $h_{b}$ along the procedure introduced at the end of Sect. 3.4. Let $\left\{Y_{i}^{*}, x_{i}^{*}\right\}_{i=1}^{n}:=\mathcal{Y}^{*}$ be a member of the pseudo sequence of samples drawn from the original sample and following the same distribution. Then, to test $H_{0}^{*}: m(x)-m_{S}(x)=\hat{m}(x)-\hat{m}_{S}(x)$, the analogs to $\tau_{1}$ and $\tau_{3}$ would be

$$
\begin{gathered}
\frac{1}{n} \sum_{i=1}^{n}\left[\left\{\hat{m}\left(X_{i}\right)-\hat{m}_{S}\left(X_{i}\right)\right\}-\left\{\hat{m}^{*}\left(X_{i}\right)-\hat{m}_{S}^{*}\left(X_{i}\right)\right\}\right]^{2}, \\
\frac{1}{n} \sum_{i=1}^{n}\left[\frac{1}{n} \sum_{j=1}^{n} \mathbf{K}_{k}\left(X_{i}-X_{j}^{*}\right)\left\{Y_{j}^{*}-\hat{m}_{S}^{*}\left(X_{j}^{*}\right)\right\}\right. \\
\left.-\mathbf{K}_{k}\left(X_{i}-X_{j}\right)\left\{Y_{j}-\hat{m}_{S}\left(X_{j}\right)\right\}\right]^{2}
\end{gathered}
$$

respectively, always neglecting the possible weighting by $w(\cdot)$ for brevity. Here, $\hat{m}^{*}$, $\hat{m}_{S}^{*}$, and $\hat{e}_{i}^{*}$ refer to estimates obtained from sample $\mathcal{Y}^{*}$. As this exercise was computationally rather expensive, we draw only $L=100$ samples $\mathcal{Y}^{*}$ and repeated the bootstrap test as before, but with the statistics (16) and (17) for all $\gamma$, and fixed $k=k_{6}:=k_{1}+5\left(k_{P}-k_{1}\right) /(P-1)$. We found that this procedure worked reasonably well for test $\tau_{3}$. However, for both $\tau_{1}$ and $\tau_{3}$ this method somewhat underestimates the real rejection level. Interestingly, this method indicates that the real rejection level decreases for increasing $\gamma$; this is consistent with asymptotic theory, but unfortunately not always with the practice. In our simulations for $h_{-\delta}=5 h$, this method recommends for $\tau_{3}$ (given $k_{6}$ ) to take $\gamma \geq 5$ when the real data-generating process is the null model, and $\gamma \geq 8$ when we draw the data from the alternative-recall that in practice we do not dispose of any information whether we are in $H_{0}$ or $H_{1}$. The predicted rejection level for $\tau_{1}$ is below $1 \%$. The real rejection levels (the power) for the different $\gamma$ can be seen in Fig. 3; they confirm that these recommendations are not bad. For $h_{-\delta}=6 h$, the method gives no clear recommendation as it predicts for $\tau_{3}\left(k_{6}\right)$ a rejection level of about $2 \%$ for all $\gamma$, and below $1 \%$ for $\tau_{1}\left(k_{6}\right)$. Again, comparing 
these predictions with Figure 4 gives some hope that the procedure may work. We guess that also for $\tau_{2}$ and $\tau_{4}$ some analogs could be constructed.

Finally, let us comment on our findings when doing subsampling instead of bootstrapping. Still focusing on the nominal level $\alpha=5 \%$, we found that $\tau_{1}$ never rejected except for $k_{\max }$ and $N \leq 0.4 n$ but without having power. Also $\tau_{2}$ was too conservative and rejected under $H_{0}$ even more often than under $H_{1}$, whereas $\tau_{3}$ held the level only for very large $k$, depending on subsample size $N$. Finally, $\tau_{4}$ showed reasonable performance for $0.6 n \leq N \leq 0.6 n$ and $k_{\max } \geq k \geq k_{7}$. Here, none of the $k$-adaptive versions worked well. To summarize, the interplay between the choice of $k$ and $N$ seems to be even more crucial than the interplay between the choice of $k$ and $h_{b}$ in the bootstrap.

\section{Conclusions}

We discuss the choices of all "parameters" a practitioner has to make when facing a smoothing-based specification test, in particular when the null hypothesis is non- or semiparametric. We have set parameters in quotation marks because we refer here also to questions like how to generate bootstrap errors, etc. For illustration, we have chosen the problem of testing additivity as this topic is well studied and many statistics have been proposed. We concentrate here on the kernel-based methods, but it is clear that for other smoothers the choice and size problems are similar.

Typically, for the null model, the practitioner has either a clear idea about the smoothness he wants to impose or he simply will go for a data-driven bandwidth $h$, cross-validation being then the most popular one. However, when bootstrap methods are used to estimate the critical value, then the bandwidth $h_{b}$ to pre-estimate the null model is crucial for the correct size and reasonable power of the test. In most cases, the asymptotic theory says that $h / h_{b}$ has to go to zero, but our simulations reveal that this choice problem is much more involved in practice.

The choice of smoothness of the alternative and test depends on $k$. There exists some literature on adaptive testing tackling exactly the problem of choosing $k$ by maximizing the power. We have reviewed different procedures, but implemented only for $\tau_{4}$ three $\mathrm{k}$-adaptive tests, namely, GL, HS, and its purely bootstrap-based version HSB. For $\tau_{1}, \tau_{2}$, and $\tau_{3}$, we implemented only the always easily available HSB version of the statistics. As we found, the GL method to be quite promising, it would be interesting how it can be extended to a broad variety of statistics. It could also be interesting to study extensions and performance of the method of Gao and Gijbels (2008) to nonand semiparametric null hypotheses.

As already mentioned in the context of choosing $h_{b}$, a main problem is the bootstrap and its size distortion in practice when the sample size is small or moderate. Concerning the residuals and wild bootstrap procedure, we concentrated on bootstrap residuals taken under the null hypothesis for reasons discussed in detail. Further, our findings confirmed the ones of Davidson and Flachaire (2008) saying that often a simpler procedure outperforms theoretically more efficient ones. We only got reasonable results for a simple wild bootstrap where the new bootstrap residual is the product of a $N(0,1)$ random variable times the residual under $H_{0}$. 
It is obvious that the proper choice of the different smoothing parameters in nonparametric specification testing is essential for size and power. These various choice problems are complex, and most of the so far known methods are computationally rather expensive. Unfortunately, this problem is typically not carefully addressed when a new test is proposed in the econometric literature. Moreover, in the existing literature, little attention has been paid to this crucial problem at all. The proposal of applying an idea borrowed from subsampling to obtain a $h_{b}$ that holds the nominal size seems to be a promising one. It entails, however, another computational expensive outer loop.

Acknowledgments The author acknowledges the very helpful discussion of the anonymous referees and the editors, which has improved the paper a lot. Further thanks goes to Jorge Barrientos, Holger Dette, Juan Rodriguez-Poo, Vladimir Spokoiny, Michael Wolf, and the participants of the seminars at the Universities of Galicia, Spain. The author acknowledges funding from the Swiss National Science Foundation, project 100018-140295, and the Spanish MCyT, project MTM2008-03010.

\section{References}

Barrientos J, Sperlich S (2010) The size problem of bootstrap tests when the null is non- or semiparametric. Revista Colombiana de Estadística 33:307-319

Cao-Abad R (1993) Bootstrapping the mean integrated squared error. J Multivar Anal 45:137-160

Cao-Abad R, González-Manteiga W (1993) Bootstrap methods in regression smoothing. Nonparametr Stat 2:379-388

Chen SX, Gao J (2007) An adaptive empirical likelihood test for parametric time series regression models. J Econom 141:950-972

Claeskens G, Van Keilegom I (2003) Bootstrap confidence bands for regression curves and their derivatives. Ann Stat 6:1852-1884

Davidson R, Flachaire E (2008) The wild bootstrap, tamed at last. J Econom 146:162-169

Delgado MA, Rodriguez-Poó JM, Wolf M (2001) Subsampling cube root asymptotics with an application to Manski's MSE. Econ Lett 73:241-250

Dette H, von Lieres und Wilkau C, Sperlich S (2005) A comparison of different nonparametric method for inference on additive models. Nonparametr Stat 17:57-81

Duong T, Hazelton M (2003) Plug-in bandwidth matrices for bivariate kernel density estimation. Nonparametr Stat 15:7-30

Eubank RL, Ching-Shang L, Wang S (2005) Testing lack of fit of parametric regression models using nonparametric regression techniques. Statistica Sinica 15:135-152

Fahrmeir L, Kneib T, Lang S (2004) Penalized structured additive regression: a Bayesian perspective. Statistica Sinica 14:731-761

Fahrmeir L, Kneib T, Konrath S (2010) Bayesian regularisation in structured additive regression: a unifying perspective on shrinkage, smoothing and predictor selection. Stat Comput 20:203-219

Gao J, Gijbels I (2008) Bandwidth selection in nonparametric kernel testing. J Am Stat Assoc 484: 1584-1594

Gao J, Gijbels I, Van Bellegem S (2008) Nonparametric simultaneous testing for structural breaks. J Econometr 143:123-142

González-Manteiga W, Martínez-Miranda MD, Pérez-González A (2004) The choice of smoothing parameter in nonparametric regression through wild bootstrap. Comput Stat Data Anal 47:487-515

González-Manteiga W, Crujeiras RM (2013) An updated review of goodness-of-fit tests for regression models. Test Forthcom 22:361-411

Gozalo PL, Linton OB (2001) Testing additivity in generalized nonparametric regression models with estimated parameters. J Econometr 104:1-48

Guerre E, Lavergne P (2005) Data-driven rate-optimal specification testing in regression models. Ann Stat 33:840-870

Härdle W, Mammen E (1993) Comparing nonparametric versus parametric regression fits. Ann Stat 21:1926-1947 
Härdle W, Marron JS (1991) Bootstrap simultaneous bars for nonparametric regression. Ann Stat 19: $778-796$

Hart JD (1997) Nonparametric smoothing and lack-of-fit tests. Springer, New York

Hastie TJ, Tibshirani RJ (1990) Generalized additive models. Chapman \& Hall, London

Hengartner NW, Sperlich S (2005) Rate optimal estimation with the integration method in the presence of many covariates. J Multivar Anal 95:246-272

Horowitz JL, Spokoiny V (2001) An adaptive, rate-optimal test of parametric mean-regression model against a nonparametric alternative. Econometrica 69:599-631

Ibragimov IA, Khasminski RZ (1981) Statistical estimation; asymptotic theory. Springer, Heidelberg

Ingster YI (1982) Minimax nonparametric detection of signals in white Gaussian noise. Probl Inf Transm 18:130-140

Ingster YI (1993) Asymptotically minimax hypothesis testing for nonparametric alternatives $I, I I, I I I$. Math Methods Stat 2:85-114

Jones MC, Davies SJ, Park BU (1994) Versions of kernel type regression estimators. J Am Stat Assoc 89:825-832

Kallenberg WCM, Ledwina T (1995) Consistency and Monte-Carlo simulations of a data driven version of smooth goodness-of-fit tests. Ann Stat 23:1594-1608

Kim W, Linton OB, Hengartner N (1999) A computationally efficient oracle estimator of additive nonparametric regression with bootstrap confidence intervals. J Comput Gr Stat 8:278-297

Köhler M, Schindler A, Sperlich S (2013) A review and comparison of bandwidth selection methods for kernel regression. Int Stat Rev 74:215-233

Kulasekera KB, Wang J (1997) Smoothing parameter selection for power optimality in testing of regression curves. J Am Stat Assoc 438:500-511

Ledwina T (1994) Data-driven version of Neyman's smooth test of fit. J Am Stat Assoc 89:1000-1005

Linton OB, Nielsen JP (1995) A kernel method of estimating structured nonparametric regression based on marginal integration. Biometrika 82:93-101

Mammen E, Linton OB, Nielsen JP (1999) The existence and asymptotic properties of a backfitting projection algorithm under weak conditions. Ann Stat 27:1443-1490

Mammen E, Park B (2005) Bandwidth selection for smooth backfitting in additive models. Ann Stat 33:1260-1294

Neumann MH, Polzehl J (1998) Simultaneous bootstrap confidence bands in nonparametric regression. J Nonparametr Stat 9:307-333

Neumeyer N, Sperlich S (2006) Comparison of separable components in different samples. Scand J Stat 33:477-501

Nielsen JP, Sperlich S (2005) Smooth backfitting in practice. J R Statl Soc B 67:43-61

Politis DN, Romano JP, Wolf M (1999) Subsampling. Springer series in statistics. Springer, Punta Gorda

Roca-Pardiñas J, Sperlich S (2007) Testing the link when the index is semiparamtric: a comparison study. Comput Stat Data Anal 12:6565-6581

Rodriguez-Póo JM, Sperlich S, Vieu P (2012) And adaptive specification test for semiparametric models. Economet Theory 29:483-523

Rosenblatt M (1969) Conditional probability density and regression estimators. Multivar Anal II:25-31

Severance-Lossin E, Sperlich S (1999) Estimation of derivatives for additive separable models. Statistics 33:241-265

Sperlich S, Linton OB, Härdle W (1999) Integration and backfitting methods in additive models: finite sample properties and comparison. Test 8:419-458

Spokoiny V (1998) Adaptive and spatially adaptive testing of a nonparametric hypothesis. Math Methods Stat 7:245-273

Su L, White H (2010) Testing structural change in partially linear models. Econometr Theory 26:1761-1806

Su L, Ullah A (2013) A nonparametric goodness-of-test-based test for conditional heteroskedasticity. Econometric Theory 29:187-212

Su L, Tu Y, Ullah A (2013) Testing additive separability of error term in nonparametric structural models. Econometr Rev (forthcoming)

Su L, Murtazasvilli I, Ullah A (2013) Local linear GMM estimation of functional coefficient IV models with an application to estimating the rate of return to schooling. J Bus Econ Stat 31:184-207

Vieu P (1993) Bandwidth selection for kernel regression: a survey. In: Härdle W, Simar L (ed) Computer intensive methods in statistics, statistics and computing 1, Physica, Berlin, pp 13-149 
Wood SN (2008) Fast stable direct fitting and smoothness selection for generalized additive models. J R Stat Soc B 70:495-518

Zhang C (2003) Calibrating the degrees of freedom for automatic data smoothing and affective curve checking. J Am Stat Assoc 98:609-629

Zhang C (2004) Assessing the equivalence of nonparametric regression tests based on spline and local polynomial smoothers. J Stat Plan Inference 126:73-95

Zheng X (1996) A consistent test of a functional form via nonparametric estimation techniques. Journal of Econometrics 75:263-289 